\title{
Escape through a time-dependent hole in the doubling map
}

\author{
André L. P. Livorati, ${ }^{1,2,{ }^{*}}$ Orestis Georgiou, ${ }^{2}$ Carl P. Dettmann, ${ }^{2}$ and Edson D. Leonel ${ }^{3}$ \\ ${ }^{1}$ Instituto de Física, IFUSP, Universidade de São Paulo, USP Rua do Matão, Tr.R 187, Cidade Universitária, 05314-970, São Paulo, SP, Brazil \\ ${ }^{2}$ School of Mathematics, University of Bristol, Bristol BS8 1TW, United Kingdom \\ ${ }^{3}$ Departamento de Física, UNESP, Universidade Estadual Paulista, Av. 24A, 1515, Bela Vista, 13506-900, Rio Claro, SP, Brazil
}

(Received 25 December 2013; published 23 May 2014)

\begin{abstract}
We investigate the escape dynamics of the doubling map with a time-periodic hole. Ulam's method was used to calculate the escape rate as a function of the control parameters. We consider two cases, oscillating or breathing holes, where the sides of the hole are moving in or out of phase respectively. We find out that the escape rate is well described by the overlap of the hole with its images, for holes centered at periodic orbits.
\end{abstract}

DOI: 10.1103/PhysRevE.89.052913

PACS number(s): 05.45.Pq, 05.45.Tp

\section{INTRODUCTION}

A recent problem of interest among both physicists and mathematicians is the study of dynamical systems with holes [1]. Escape occurs when trajectories enter some predefined subset of the phase space called a hole. This "leaking" of trajectories can happen in bounded $[2,3]$ as well in unbounded domain systems [4-6]. A natural observable which allows the study of statistical properties of this escape, is the probability (given a suitable distribution of initial conditions) that an orbit does not escape until a time $n$. This raises the natural question of the decay rate of $\rho(n)$. The most important aspect of this analysis is that the escape rate is very sensitive to the system dynamics. For strongly chaotic systems the decay is typically exponential [7], while for systems that present mixed phase space (e.g., elliptic islands and a chaotic sea), the decay can be slower, presenting a mix of exponential with a power law $[8,9]$ or stretched exponential decay [6]. Indeed, when a nonexponential decay is observed, the dynamics would require a long-range correlation, such as a consequence of stickiness influence [8]. An equally important aspect is that the escape rate can have a strong dependence on the position and size of the hole [3,10]. Applications of leaking systems can be found in a great variety of fields, including plasmas $[11,12]$, acoustics [13,14], optics [15,16], and fluids [17], among others (see Ref. [1] for a recent review).

While in most mathematical formulations of leaking systems the hole is static (and typically small relative to the phase space), in this paper we undertake a new approach and study escape through a time-dependent hole. Namely, we propose and investigate the escape properties of a chaotic leaking system where the hole position and hole size vary with time. Motivation for studying such problems can be traced back to the early 1950s concerning Moshinsky's shutter problem of "diffraction in time" [18]. More recent applications can by found in quantum mechanics $[19,20]$ and in atom optics and ultra-cold atoms experiments [21-24]. Further motivation for studying time-dependent holes stems from chemical reactions and hydrodynamical flows (see, for example, the blinking vortex system [25]).

We restrict our investigations of time-dependent holes to the well-studied and understood case of the open doubling

*livorati@usp.br map (defined in the next section) [3,26-32]. What is particularly attractive about this map is that it is uniformly expanding with a uniform invariant density distribution and also has a well-understood structure of periodic orbits due to the correspondence between the dynamics and the binary representation of phase space points. We aim to understand the role of these periodic orbits in the case of escape through a time-dependent hole, once they can play an important role in other dynamical systems $[33,34]$. To this end, we present extensive numerical investigations and construct accurate analytical predictions for $\rho(n)$.

The remainder of the paper is organized as follows: In Sec. II we describe how the time-dependent hole is introduced and some properties concerning the escape rate and the periodic orbits. The numerical and analytical results are shown in Sec. III. Finally some final remarks and conclusions are drawn in Sec. IV.

\section{THE MAPPING, PROPERTIES, AND TIME-DEPENDENT HOLE}

The dynamical system under study here is the doubling map modulo one, also known as the Bernoulli shift, represented by the recurrence relation

$$
x_{n+1}=2 x_{n} \quad \bmod (1) .
$$

The phase space is shown in Fig. 1. Because of the simple nature of the dynamics when we consider the binary notation [3], it is easy to categorize the dynamics based on the initial condition. If the initial condition is irrational, which are almost all points in the unit interval, the dynamics is nonperiodic, which follows directly from the definition of an irrational number as one with a nonrepeating binary expansion. However, if $x_{0}$ is rational, the image of $x_{0}$ contains a finite number of distinct values within the interval $[0,1)$ and the forward orbit of $x_{0}$ is eventually periodic, with period equal to the period of the binary expansion of $x_{0}$. In particular, if the initial condition is a rational number with a finite binary expansion of $k$ bits, then after $k$ iterations the iterates reach the fixed point 0 ; if the initial condition is a rational number with a $k$-bit transient $(k \geqslant 0)$ followed by a $p$-bit sequence $(p>1)$ that repeats itself infinitely, then after $k$ iterations the iterates reach a cycle of length $p$. Thus cycles of all lengths are possible [3]. Another way of representing the periodic 


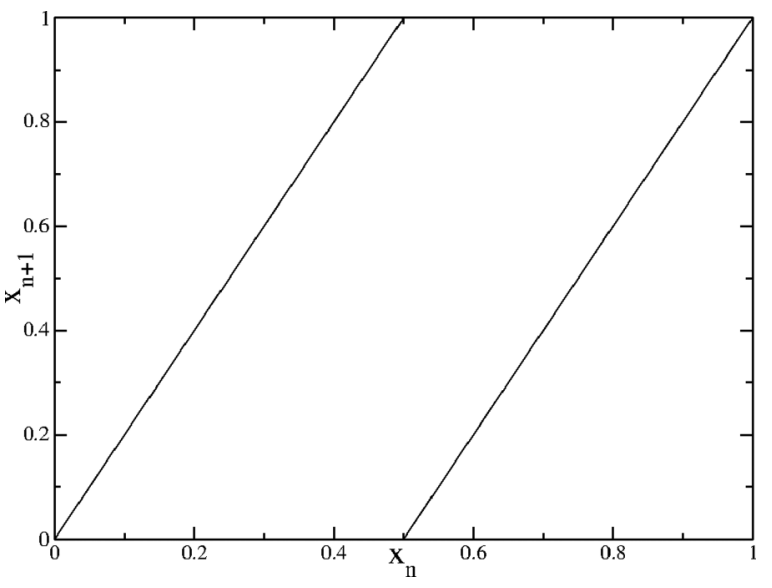

FIG. 1. Phase space for the doubling map.

orbits is

$$
x_{0}=\frac{q}{\left(2^{p}-1\right)},
$$

where $q \in \mathbb{Z}$ and $p$ is the period of the periodic orbit. So, for example, if we choose an initial condition with $q=8$ and $p=4$, it would be a period- 4 orbit with dynamics evolving as $8 / 15 \rightarrow 1 / 15 \rightarrow 2 / 15 \rightarrow 4 / 15 \rightarrow 8 / 15$.

Once the main properties of the mapping are known, let us now introduce the escape in the dynamics, by considering a time-dependent hole, i.e., a hole whose position and/or size is varying periodically.

Define the closed domain map as

$$
f:[0,1] \rightarrow[0,1],
$$

where $f$ is the application of the mapping in Eq. (1). The open map is given by

$$
\hat{f}:[0,1] \backslash H_{n} \rightarrow[0,1],
$$

where $H_{n} \subset[0,1]$ is the hole at time $n$. Points within the hole are deemed to escape and are not considered further.

We set a mean fixed position for the hole to oscillate, $\bar{x}$, that could be in the neighborhood of a short periodic orbit, or even the periodic orbit itself. Choosing the mean position around a periodic orbit allows us to compare the results we obtain with the results already known in the literature for the fixed hole position [10,30-32].

So, once the mean position is set up, we may define the hole size. Since this value should vary with time, we can work with an average size of the hole, which we will name $\bar{h}$. So two fixed positions for the hole to oscillate were set. These positions represent the hole boundaries, and we define them as the hole boundary at the right $h_{r}$ and the hole boundary at the left $h_{l}$; and they are set as

$$
\begin{aligned}
& h_{r}=\bar{x}+\bar{h} / 2 \\
& h_{l}=\bar{x}-\bar{h} / 2 .
\end{aligned}
$$

The expressions given in Eq. (5), are saying that we have a hole with size $\bar{h}$, and its position is symmetric centered in $\bar{x}$.

When we introduce the time dependence on the hole, we must deal with a discrete recurrence relation $(n)$, and not a continuous one as time $(t)$. So, with a periodic oscillation, the

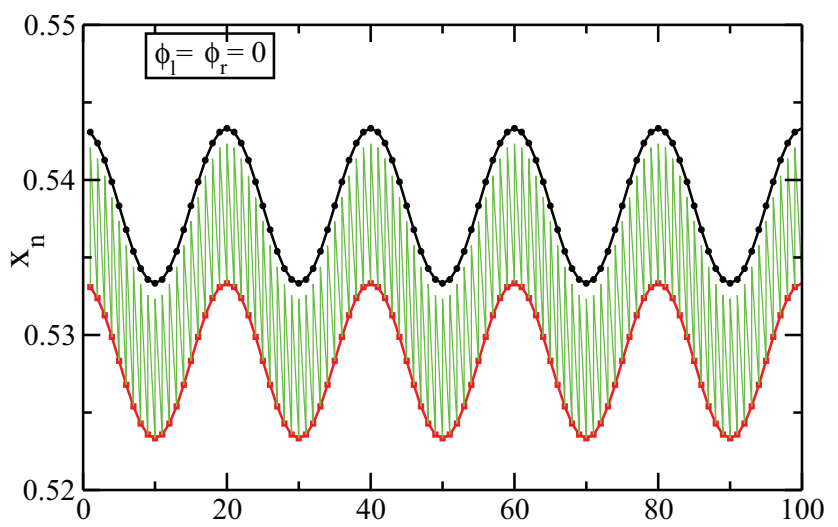

(a)

n

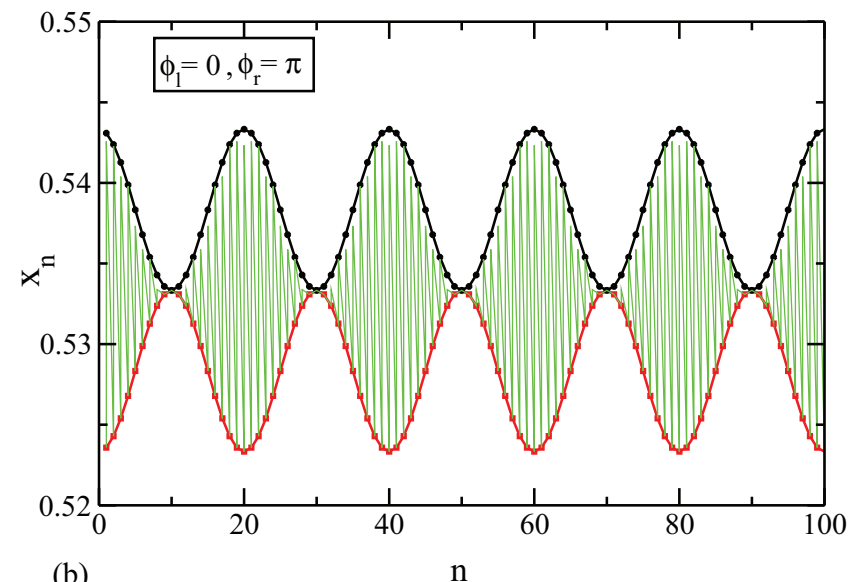

(b)

FIG. 2. (Color online) Evolution of the time-dependent hole for two combinations of the initial phases for a value of $\tau=20$ and a mean value around a period-4 orbit located in (8/15). In (a) both $h_{l}(n)$ and $h_{r}(n)$ are in phase with each other, so the size of the hole is kept constant during the dynamics and only its position is moving. In (b), $h_{l}(n)$ and $h_{r}(n)$ are not in phase, so the size of the hole is varying, but the average hole $\bar{h}$ is constant by period of oscillation. $h_{l}(n)$ is represented by the black line with bullets, and $h_{r}(n)$ shown as the red line with squares. The green dashed lines are the escape allowed region.

expressions of Eq. (5) can be written as

$$
\begin{aligned}
& h_{r}(n)=h_{r}+\epsilon \cos \left(\omega n+\phi_{r}\right) \\
& h_{l}(n)=h_{l}+\epsilon \cos \left(\omega n+\phi_{l}\right),
\end{aligned}
$$

where $\epsilon$ is the amplitude of oscillation of the holes, $\omega=(2 \pi / \tau)$ is the frequency of oscillation, and $\phi_{l}$ and $\phi_{r}$ are the initial phases of oscillation. The behavior of each boundary of the hole according to as $n$ evolves is illustrated in Fig. 2.

The value of the phases $\phi_{l}$ and $\phi_{r}$, in particular, whether they are equal or not, will influence the value of the amplitude of oscillation $\epsilon$, that may be chosen in order to keep the left and right boundaries of the hole defined in Eq. (6) in the $x$-axis domain. Figure 2 shows how the hole would oscillate as $n$ evolves for a mean position at a period-4 orbit. If $\phi_{l}$ and $\phi_{r}$ are in phase as shown in Fig. 2(a), the position of the hole is moving as $n$ evolves, but it remains with the same size; here we have no limit for the value of $\epsilon$, provided that is inside the 
domain of the doubling map. However, if $\phi_{l}$ and $\phi_{r}$ are not in phase, as shown in Fig. 2(b), the hole size is moving, in a breathing way. Here we have the limit breathing case, that is, $\epsilon \leqslant \bar{h} / 2$. In this limit, we have a tangency between $h_{l}(n)$ and $h_{r}(n)$, when the period is complete, where the hole vanishes momentarily. If we go beyond this limit, there would be some prohibited regions for the escape, and we are not considering this case in this paper.

\section{METHODS, RESULTS, AND DISCUSSIONS}

In this section we will present analytical and numerical methods to evaluate the escape through the time-dependent hole. We investigate how the escape rate varies with the control parameters $\epsilon, \tau$ and the combination of initial phases $\phi_{l}$ and $\phi_{r}$. We also make frequent comparison with the static hole case.

\section{A. Ulam's method and escape rates}

We make use of Ulam's method to calculate the escape rates for the time-dependent hole. Ulam's method is a numerical scheme for approximating invariant densities of dynamical systems that can be made rigorous [35-39]. The phase space is partitioned into connected sets, and an interset transition matrix is computed from the dynamics; an approximate invariant density is read off as the leading left eigenvector of this matrix. When a hole in phase space is introduced, one instead searches for conditional invariant densities and their associated escape rates [36-43]. In other words, we divide the space $X$ into a fine partition $X_{i}$ and assume that the probability of a transition from $i$ to $j$ is given by the proportion of $X_{i}$ that is mapped into $X_{j}$, that is,

$$
\rho_{i j}=\frac{\left|X_{i} \cap \hat{f}^{-1}\left(X_{j}\right)\right|}{\left|X_{i}\right|} .
$$

If we consider the static hole case, given that the doubling map has exponential decay of correlations, it seems clear that the survival probability should be exponential exponential, with a rate depending on the hole position and size, as studied previously [3,30-32]. For the same doubling map, the escape rate is

$$
\gamma=-\lim _{n \rightarrow \infty} \frac{1}{n} \ln \rho(n) .
$$

For a time-dependent hole, we may find an exponential decay related as suggested by Eq. (8), but the time dependence can also have a superimposed periodic oscillation, as discussed in Sec. III C.

We are considering the two different cases of initial phases of $\phi_{l}$ and $\phi_{r}$, as shown in Fig. 2 in a separate way. Initial conditions used to calculate the survival probability for both kinds of holes were chosen equally split in the interval $[0,1]$. Let us first address the case $\phi_{l}=\phi_{r}$ where only the hole position is moving and its size $\bar{h}$ is kept constant. Figure 3 shows how these escape rates behave for some different average hole sizes, different values of amplitude of oscillation $\epsilon$, and different values of $\tau$. For this figure, we decided to keep the mean value of the hole position $\bar{x}=8 / 15$. In later sections, we address other mean values of the hole $\bar{x}$. As expected in Figs. 3(a) and 3(c) with an average hole of $\bar{h}=0.1$, we
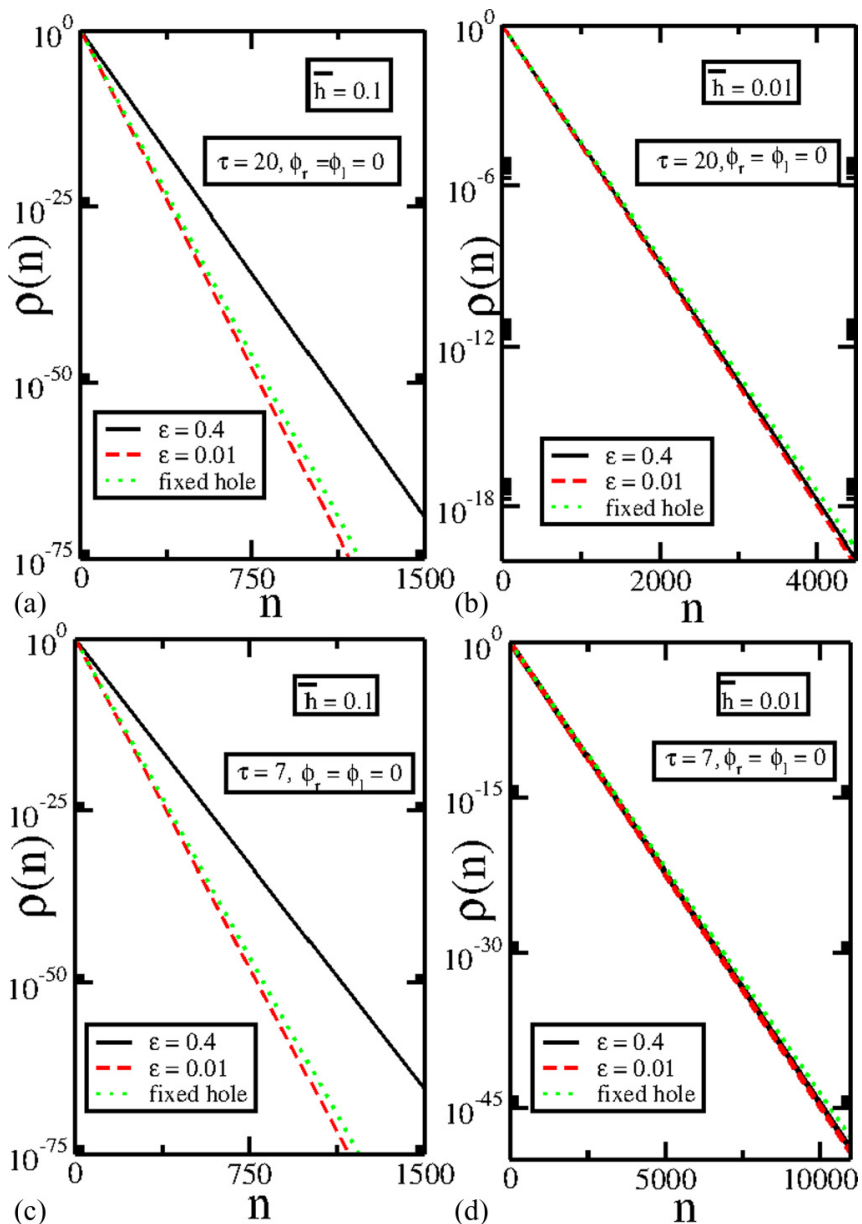

FIG. 3. (Color online) Survival probability curves for different values of the average moving hole with equal initial phases. In (a) and (b) we have $\tau=20$, and in (c) and (d) $\tau=7$. Also, we ranged the value of the amplitude of oscillation $\epsilon$ and made a comparison with the fixed hole case. Depending of the combination of $\epsilon$ and $\tau$ we may have faster or slower escape.

have a much faster decay than in Figs. 3(b) and 3(d), where the average hole is $\bar{h}=0.01$. However, one can also notice that depending on the combination of $\epsilon$ and $\tau$ parameters, we may have a faster or slower escape, as shown in Figs. 3(a) and 3(c), for a bigger value of $\epsilon=0.4$ which basically contains the whole domain of the doubling map. Also, one can notice that the labels of the $\rho(n)$ axis in Fig. 3 are very small. This precision is a result of the application of Ulam's method, which can be very accurate depending on the number of partitions. In Table I one may find the value of the escape rate for some combinations of values of $\tau$ and $\epsilon$.

One can see that the value of $\gamma$ is proportional to the average hole size, which would be roughly expected according to Refs. [3,10,29,32], but there are also significant changes, depending on the combinations of the control parameters. In order to understand better how the escape rate varies with $\epsilon$ and $\tau$, we plotted the value of the escape rate $\gamma$ versus $\tau$ for several values of $\epsilon$, as shown in Fig. 4. Here we keep the average hole size $\bar{h}=0.01$ and consider three different mean values for the hole to oscillate $(\bar{x})$ about three different periodic orbits. We see that for small values of $\tau$, there is 
TABLE I. Value of the escape rate $\gamma$ for a combination of $\epsilon$ and $\tau$ for two different hole sizes.

\begin{tabular}{lrcc}
\hline \hline $\bar{h}$ & \multicolumn{1}{c}{$\tau$} & $\epsilon$ & $\gamma$ \\
\hline 0.1 & 7.0 & 0.4 & $0.1004784(1)$ \\
0.1 & 7.0 & 0.01 & $0.1468975(5)$ \\
0.1 & 20.0 & 0.4 & $0.1061757(6)$ \\
0.1 & 20.0 & 0.01 & $0.1473164(5)$ \\
0.1 & 0.0 & Fixed hole & $0.1418285(1)$ \\
0.01 & 7.0 & 0.4 & $0.01024197(3)$ \\
0.01 & 7.0 & 0.01 & $0.01030896(5)$ \\
0.01 & 20.0 & 0.4 & $0.01020202(1)$ \\
0.01 & 20.0 & 0.01 & $0.0103262(1)$ \\
0.01 & 0.0 & Fixed hole & $0.0100119(1)$ \\
\hline \hline
\end{tabular}

a large variation in the escape rate for all values of the mean hole, considering some high values of $\epsilon$ (say, above $\epsilon=0.001$, which is $10 \%$ of the average hole size). These fluctuations can be explained: once $\epsilon$ is big enough and $\tau$ small, the hole is moving up and down really fast as the dynamics evolves, and during this movement, it can intercept several different periodic orbits, which may be one of the explanations for the variation of the escape rate. However, for high values of $\tau$, the escape rate stays almost constant. This should be expected, since for high values of $\tau$, the hole takes a time much longer then other time scales in this problem to change its position. Also, if we look for the curves with small values of $\epsilon$, one can see that they stay in a constant regime for all values of $\tau$, as once $\epsilon \rightarrow 0$, the moving hole starts to behave like a fixed hole. Comparing the limit of $\epsilon \rightarrow 0$ for Figs. 4(a)-4(c), we can see that the plateau where the escape rate establish itself changes, when we consider a different periodic orbit. Indeed, as shown previously [3,10,31,32] the escape occurs faster through a hole which contains long periodic orbits and is slower if the hole contains short periodic orbits. Recall that, according to the literature $[3,10,32]$, the escape rate through a small hole covering a short periodic orbit is approximately given by

$$
\gamma=\bar{h}\left(1-\Lambda^{-1}\right)
$$

where $\Lambda=2^{p}$ and $p$ is the period of the periodic orbit, represented by dashed lines for all the periodic orbits in Fig. 4 . We also observe higher order corrections to the expression presented in Eq. (9). These higher order corrections are specifically detailed in Refs. [10,32], and we think that if they were taken into account together with Eq. (9), there would be a good agreement between them. Also, one could ask, by choosing a mean position for the hole to oscillate as an irrational number, if there would be any different result. We think that the results would be basically the same. The escape rate must be in somehow proportional to the hole size and present itself as an exponential decay. Perhaps a small difference would be the analytical treatment concerning the corrections related to periodic orbits, as the first order correction in Eq. (9).

\section{B. Overlap holes}

We now develop an analytic approach where both $\phi_{l}$ and $\phi_{r}$ are equal, as a function of the control parameters $\tau$ and $\epsilon$, using the overlap of the periodic orbits with the $p$ application of the
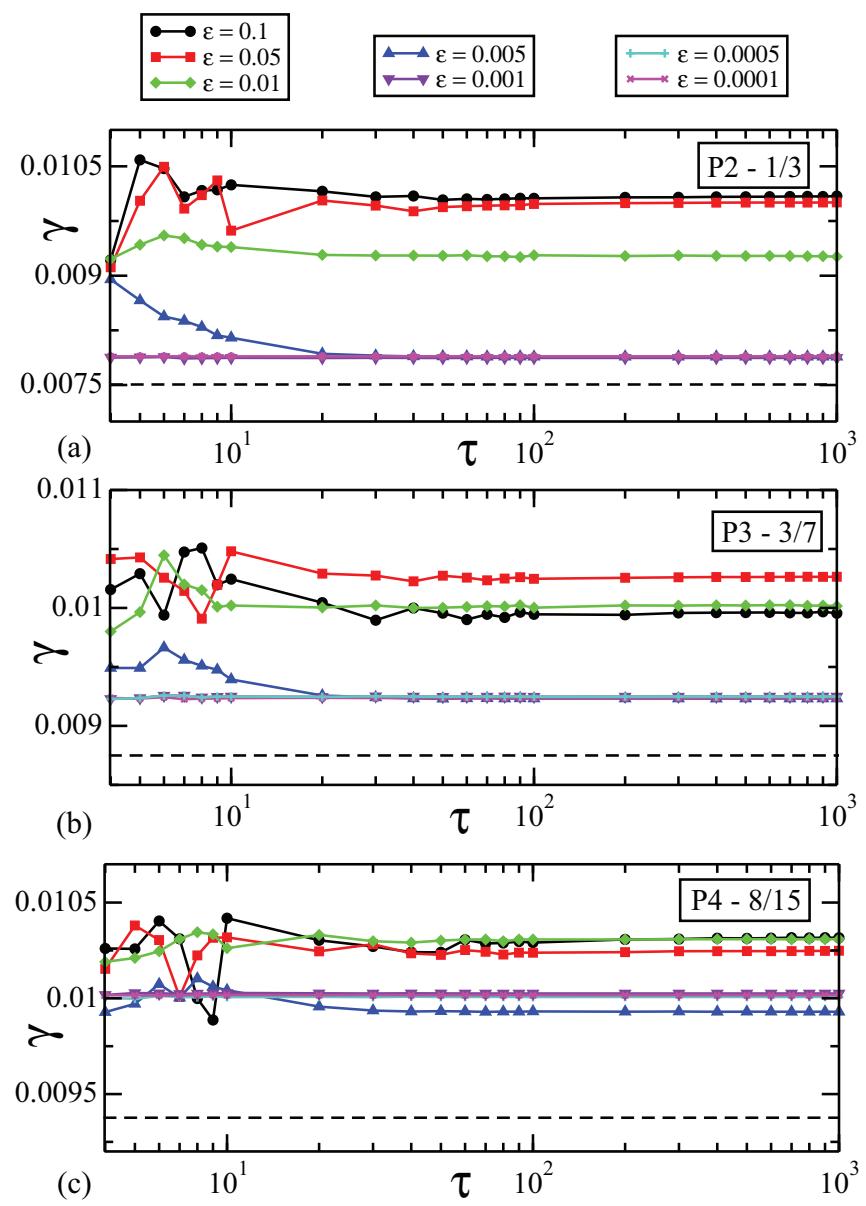

FIG. 4. (Color online) Variation of the escape rate $\gamma$ as function of $\tau$ for several values of $\epsilon$. Here the average hole is fixed at $\bar{h}=0.01$, and we considered three different periodic orbits for the mean hole position. In (a) $\bar{x}=1 / 3$, a period- 2 orbit, in (b) $\bar{x}=3 / 7$, a period-3 orbit, and in (c) $\bar{x}=8 / 15$, a period- 4 orbit. Notice that for high values of $\epsilon$, for a small $\tau$ regime, the escape rate varies a lot, and as $\tau$ increases it bends towards an almost constant regime. For the limit $\epsilon \rightarrow 0$, the escape rate behaves closer as the one expected for a fixed hole, for all values of $\tau$. The dashed lines represents the first order approximation of the fixed hole escape rate, according to Eq. (9).

mapping in Eq. (1). The motivation for this kind of attempt came from Ref. [26], where an extensive analytical analysis is made concerning the escape rate on the doubling map. What the overlap hole approach does is basically a "correction" of Eq. (9), concerning the moving hole. Considering these overlaps, we can write the escape rate as

$$
\gamma_{o h}=\bar{h}\left[1-\frac{1}{\tau} \sum_{i=0}^{\tau-1} \frac{\left|f^{p}\left(H_{i}\right) \cap H_{i+p}\right|}{\left|f^{p}\left(H_{i}\right)\right|}\right],
$$

where the index $o h$ means the overlap holes, $H_{i}$ is the hole size in the $i$ th iteration, and $H_{i+p}$ is the hole size considered on the $i+p$-th iteration.

We make a comparison of the results obtained considering the numerical simulations using Ulam's method and an analytical approach by the formula expressed in Eq. (10), for three different periodic orbits of low period and for two values of the average hole. The numerical data are represented by the 
full lines, and the analytical approach is given by the dotted lines. Although both data follow a similar behavior, as we increase the value of $\tau$, one can see in Fig. 5 that there is still a gap between the numerical data and the analytical approach. We can attribute these gaps to higher order corrections; for a fixed hole the escape rate should follow $\gamma=\bar{h}(1-\Lambda)+o(\bar{h})$, where the corrections may lie on the form $\bar{h}^{2} \ln \bar{h}$ [32]. The analytical data plotted in Fig. 5 are hence adjusted according to

$$
N\left[\gamma_{o h}(\tau)\right]=\gamma_{o h}(\tau)+\gamma_{n}(\infty)-\gamma_{o h}(\infty),
$$

where $N\left[\gamma_{o h}(\tau)\right]$ is the normalized escape rate considering the overlap holes approach, $\gamma_{o h}(\tau)$, is the analytical approach for the escape rate concerning the overlap holes according to Eq. (10), $\gamma_{n}$, is the numerical escape rate obtained by Ulam's method. The argument $\tau \rightarrow \infty$ is taken along an average between $\tau=100$ and $\tau=1000$, once the escape rate for this case is almost constant. So, with this correction, the higher order effects are taken into account, and the matching between the numerical and the analytical approach concerning the overlap holes in Eqs. (10) and (11) occurs. However, one can still see small discrepancies for small values of $\tau$, where the hole is moving too fast. These still need further investigation.

\section{Breathing hole}

Now we address the case where $\phi_{r}=0$, and $\phi_{l}=\pi$, shown in Fig. 2(b), where the hole size is in constant change. Now, the hole is increasing and decreasing in a periodic way as $n$ evolves, in a breathing way, but the average hole size is the same over the period of oscillation. Figure 6(a) shows the escape rate curve for this kind of moving hole, for some values of $\tau$ for a hole centered in a mean position of $\bar{x}=$ $8 / 14$. We can see that, in general it decays as an exponential envelope, but with a peculiarity: it decays in steps. The step obeys the period of oscillation of the moving hole, as shown in the comparison made in Figs. 6(b) and 6(c). The steps can be basically explained by this comparison. Once the hole is increasing and decreasing, the rate of orbits that escape through it varies according to its size. So, when we have a tangency between both hole sides $h_{l}$ and $h_{r}$, none of the orbits are escaping, and then we have a constant plateau of $\rho(n)$. On the other hand, when the hole is in its fully open size, we have a faster escape. Here we used the value of $\epsilon=\bar{h} / 2$, that is, the limit case for an instantaneous prohibited escape zone. If we had a smaller value for $\epsilon$, these steplike decays would be smoother, and in the limit that $\epsilon \rightarrow 0$, it would behave as a completely exponential curve decay.

An attempt for an analytical approach for the breathing hole can be made. We have that the hole is $h(n)=h_{r}(n)-h_{l}(n)$,
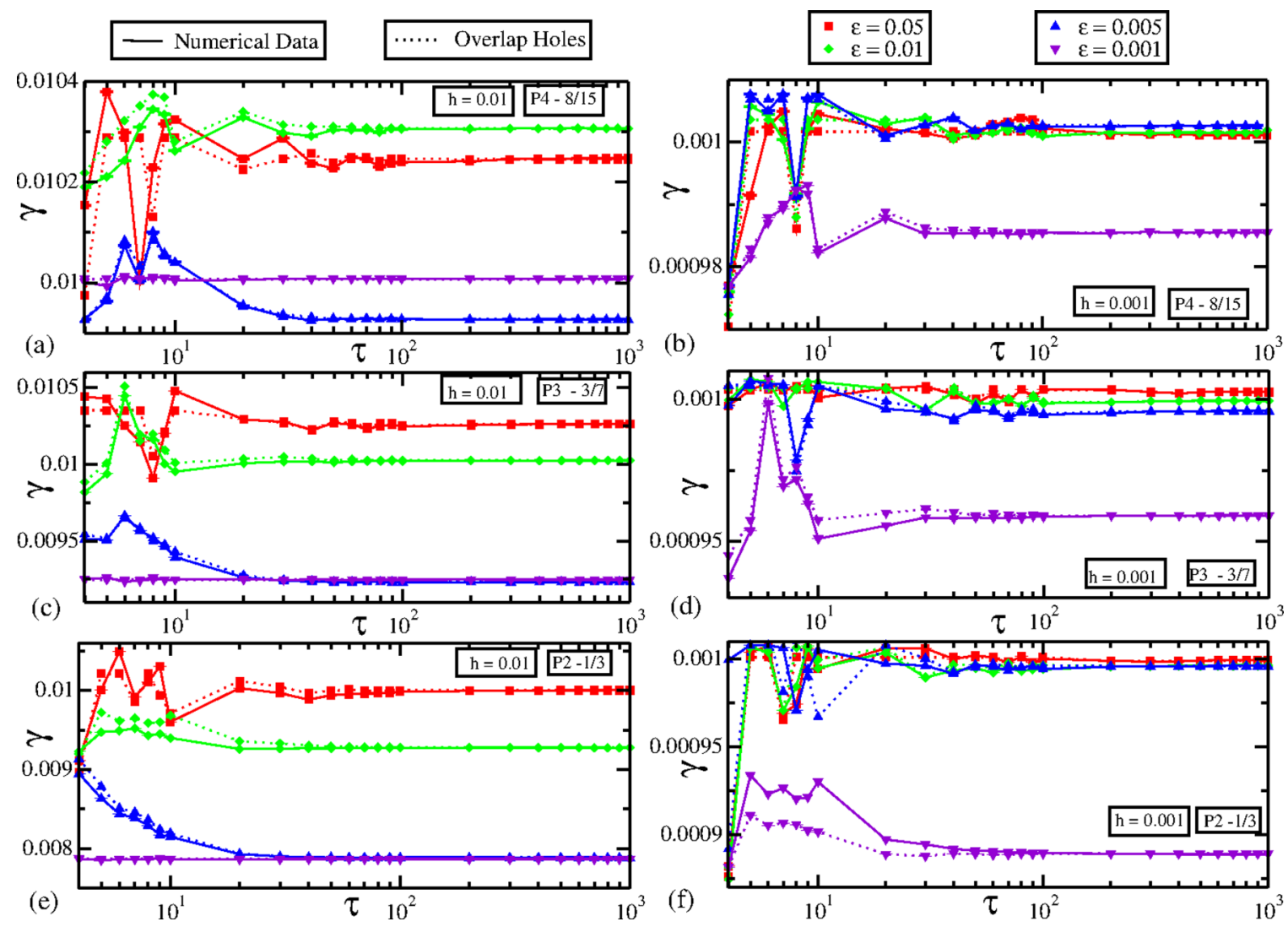

FIG. 5. (Color online) Comparison between the numerical data and the analytical approach for the overlap holes, given by Eqs. (10) and (11). In (a), (c), and (e) the average hole is $\bar{h}=0.01$, and in (b), (d), and (f) we have $\bar{h}=0.001$. Also, the numerical data are given by the full lines, and the analytical approach is given by the dotted lines. Red squares represent $\epsilon=0.05$, green diamonds $\epsilon=0.01$, blue up triangles $\epsilon=0.005$, and purple down triangles $\epsilon=0.001$. The matching is really good for high values of $\tau$, where the hole is moving slowly, but for low values of $\tau$, where the hole is moving faster, there is still a gap between them. 


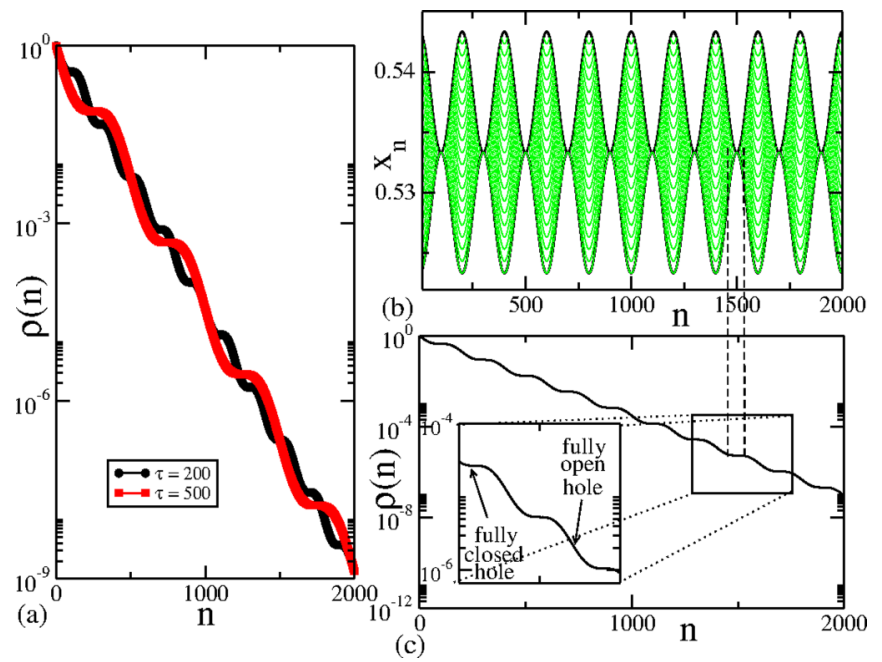

FIG. 6. (Color online) Steplike decay behavior for the probability curves when $\phi_{r}=0$ and $\phi_{l}=\pi$. We kept the average hole size constant in $\bar{h}=0.01$ and $\epsilon=0.005$, which is $\epsilon=\bar{h} / 2$. In (a) we have $\tau=200$ and $\tau=500$. One can notice that the steplike decays of the survival probability follows the period of oscillation in a exponential envelope. In (b) and (c) we have a comparison of the hole behavior with the probability decays. When the hole is fully open, we have a faster escape, and when the hole is fully closed (tangency), we have a constant plateau, where no orbits are escaping. The zoom window in (c) shows better this steplike behavior.

where $h_{r}(n)$ and $h_{l}(n)$ are given by Eq. (6), where the initial phases are $\phi_{r}=0$ and $\phi_{l}=\pi$. So we may say that the hole size obeys

$$
h(n)=\bar{h}+2 \epsilon \cos (\omega n)
$$

where $\omega=2 \pi / \tau$ and $\epsilon$ must not be bigger than $\bar{h} / 2$.

Now that the hole size is known we may propose the following expression where the decay of the survival probability as function of $n$, as shown in Fig. 6, is given by

$$
\frac{d \rho}{d n}=-\rho\{[\bar{h}+2 \epsilon \cos (\omega n)] \times P(n o h)\},
$$

where $P(n o h)$ is the probability of a point in the hole not overlapping with the hole on the $(n+p)$-th iteration, just like we did for the the moving hole case, where again $p$ is the period of the periodic orbit where the hole is centered. This probability is given by

$$
P(n o h)=\frac{\bar{h}+2 \epsilon \cos [\omega(n+p)]}{\bar{h}+2 \epsilon \cos (\omega n)} \times \frac{1}{\Lambda} .
$$

Replacing the above expression and solving the separable equation, we have the following expression:

$$
\begin{aligned}
\rho(n)= & \exp \left\{-\bar{h} n\left(1-\Lambda^{-1}\right)\right. \\
& \left.-\frac{2 \bar{h} \epsilon}{\omega}\left[\sin (\omega n)+\frac{\sin [\omega(n+p)]}{\Lambda}\right]\right\} .
\end{aligned}
$$

In the above expression, the term $\bar{h} n\left(1-\Lambda^{-1}\right)$ can be named $\overline{\gamma(n)}$. Let us do an attempt to improve this expression making use of second order approximations. If we consider a fixed hole, the escape rate considering second order effects $[26,32]$ can be given by

$$
\gamma_{\text {fixed }}=h\left(1-\Lambda^{-1}\right)+a_{p} h^{2} \ln (h),
$$

where $a_{p}$ is a constant that may depend on the $p$-periodic orbits. In order to find the $a_{p}$ value, we simulated for the several values of the fixed hole and compared the numerical result of $\gamma=-\lim _{n \rightarrow \infty} \frac{1}{n} \ln \rho(n)$ with the analytical approach of Eq. (16), and found an average value for $a_{p}: a_{2} \approx 1.812$, $a_{3} \approx 2.055$, and $a_{4} \approx 2.331$. We stress that the $p$-periodic orbits considered for the hole to be centered were the same ones used in the previous section.

In the breathing case, the hole size is in constant change as $n$ evolves, so we must assume that the escape rate is not constant either, as one can see in Fig. 6. So, according to Eqs. (12) and (16), we may set

$$
\gamma=h(n)\left(1-\Lambda^{-1}\right)+a_{p} h(n)^{2} \ln [h(n)] .
$$

Once we have an average value for the hole as $\bar{h}$, we can consider also an average over the escape rate as

$$
\bar{\gamma}=\frac{\omega}{2 \pi} \int_{0}^{\frac{2 \pi}{\omega}} \gamma d n .
$$

Replacing Eq. (12) and evaluating this average on Eq. (18), one can obtain

$$
\begin{aligned}
\bar{\gamma}= & \bar{h}\left(1-\Lambda^{-1}\right)+a_{p}\left\{\bar{h}[3 \mu-\bar{h}(3 \ln 4)]-\epsilon^{2}(2 \ln 16)\right\} \\
& -2 a_{p}\left(2 \epsilon^{2}+\bar{h}^{2}\right) \frac{\ln (\bar{h}+\mu)}{2},
\end{aligned}
$$

where $\mu=\sqrt{-4 \epsilon^{2}+\bar{h}^{2}}$. Now the steplike behavior of $\rho(n)$ can be analytically expressed by the combinations of Eqs. (15) and (19), where higher order effects are considered:

$$
\rho(n)=\exp \left\{-\bar{\gamma} n-\frac{2 \bar{h} \epsilon}{\omega}\left(\sin (\omega n)+\frac{\sin [\omega(n+p)]}{\Lambda}\right)\right\} .
$$

Figure 7 shows a comparison between the analytical (dotted lines) and the numerical data (full lines) for $\tau=200$, for three distinct periodic orbits, concerning a hole size $\bar{h}=0.01$. One can see that for the limit case $\epsilon=\bar{h} / 2$ in Fig. 7(a), the steplike decay in the exponential envelope is present according to the hole period of oscillation, and the analytical approach matches reasonably well. If we decrease the amplitude of oscillation, to $\epsilon=0.001$ as shown in Fig. 7(b), the survival probability curve presents a smooth behavior concerning the steplike decays, and the exponential envelope is more dominant in the decay. For this case of smaller $\epsilon$ there is no longer a instantaneous forbidden region in the hole evolution. However, concerning the zoom-in windows in Figs. 7(c) and 7(d), there is still a little gap between the numerical and analytical data. We believe that this gap may be due to higher order effects, which may be introduced in Eq. (17) in order to improve the analytical expression. 

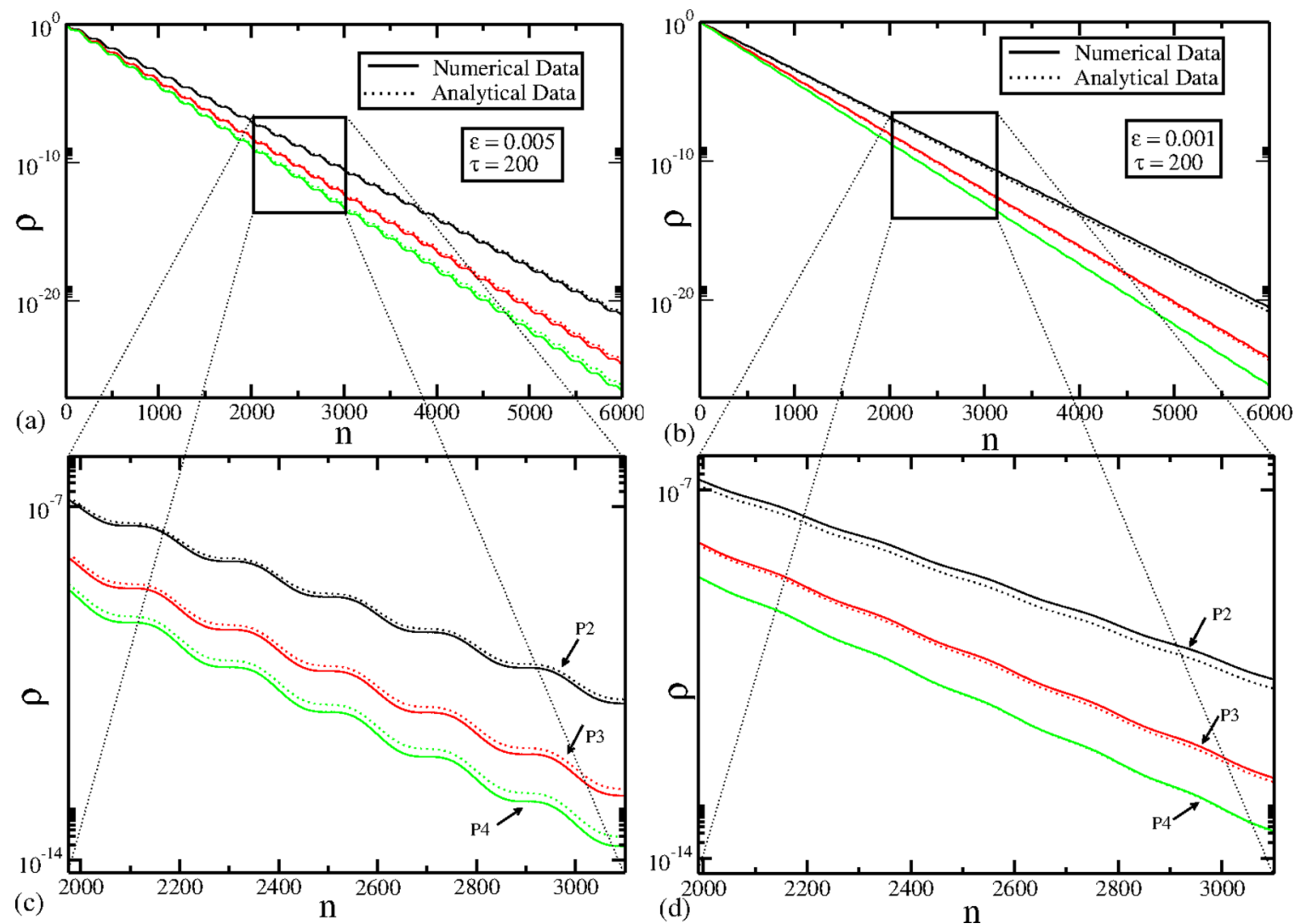

FIG. 7. (Color online) Comparison between the numerical steplike decays and the analytical approach given by Eq. (20) for $\tau=200$ and $\bar{h}=0.01$. In (a) $\epsilon=0.005$, and in (b) $\epsilon=0.001$. One can notice a remarkably good match between the numerical and the analytical data in the amplifications in (c) and (d).

\section{FINAL REMARKS AND CONCLUSIONS}

We investigated the escape dynamics of the doubling map with a time-periodic hole with amplitude and period of oscillation, $\epsilon$ and $\tau$, respectively. We considered two distinct ways for the hole to oscillate: (1) keeping the same size and changing its position and (2) breathing case. This two kinds of hole are controlled by an initial phase $\phi_{l}$ and $\phi_{r}$ introduced in the time-dependent perturbation.

Using Ulam's method to calculate the probability of escape, we found that it is basically exponential, and for case (1) it depends on the value of $\tau$ and $\epsilon$. If we had a low $\tau$, the hole is moving really fast, and we observe some fluctuations on the escape rate $\gamma$ versus $\tau$ curves. If the hole is moving more slowly, the escape rate is correspondingly more slowly varying with $\tau$. Also, for some high values of $\epsilon$, the hole can intercept many periodic orbits, which can add even more fluctuations on the escape rate, and for $\epsilon \rightarrow 0$, it reduces to a fixed hole. In an attempt to explain these fluctuations, we introduced an analytical approach related to overlap holes. We observed that the numerical data and the analytical results have excellent agreement if higher order effects are taken into account. Considering case (2), we observed that the probability decays according a steplike function in an exponential envelope, which follows the value of period of oscillation $\tau$ for the breathing hole. We set up an analytical approach for the steplike decay also considering the probability of overlap holes, and it reasonably matches with the numerical data. Also, in the limit $\epsilon \rightarrow 0$, the steplike is very smooth, and the survival probability can be expressed as an exponential law.

We emphasize that the control parameters strongly affect the escape rate, for the moving hole, considering a fast and slow moving hole, or the breathing case. As a next step, we would try to find the exactly higher order effects for the escape rate and improve the analytical expressions for both hole cases. Also, it would be interesting to see how the escape rate would vary for periodically moving holes in more complicated systems, such as with a mixed phase space, and for nonperiodic hole perturbations, for example, random ones.

\section{ACKNOWLEDGMENTS}

A.L.P.L. acknowledges $\mathrm{CNPq}$ and CAPES-Programa Ciências sem Fronteiras-CsF (0287-13-0) for financial support. E.D.L. thanks FAPESP (2012/23688-5), CNPq and CAPES, Brazilian agencies. A.L.P.L. also thanks the University of Bristol for the kind hospitality during his stay in the U.K. This research was supported by resources supplied by the Center for Scientific Computing (NCC/GridUNESP) of the São Paulo State University (UNESP). The authors are also grateful for fruitful discussions with Georgie Knight and Eduardo G. Altmann. 
[1] E. G. Altmann, J. S. E. Portela, and T. Tél, Rev. Mod. Phys. 85 869 (2013).

[2] Ying-Cheig Lai and Támas Tél, Transient Chaos: Complex Dynamics on Finite-Time Scales, Ap. Math. Sci. (Springer, New York, 2011).

[3] L. A. Bunimovich and A. Yurchenko, Isr. J. Math 182, 229 (2011).

[4] P. Gaspard and G. Nicolis, Phys. Rev. Lett. 65, 1693 (1990).

[5] P. Gaspard and D. A. Ramirez, Phys. Rev. A 45, 8383 (1992).

[6] C. P. Dettmann and E. D. Leonel, Physica D 241, 403 (2012).

[7] E. D. Leonel and C. P. Dettmann, Phys. Lett. A 376, 1669 (2012).

[8] A. L. P. Livorati, T. Kroetz, C. P. Dettmann, I. L. Caldas, and E. D. Leonel, Phys. Rev. E 86, 036203 (2012).

[9] E. G. Altmann and T. Tél, Phys. Rev. E 79, 016204 (2009).

[10] G. Keller and C. Liverani, J. Stat. Phys. 135, 519 (2009).

[11] J. S. E. Portela, I. L. Caldas, and R. L. Viana, Int. J. Bifurcat. Chaos 17, 4067 (2011).

[12] D. del-Castillo-Negrete, B. A. Carreras, and V. E. Lynch, Phys. Rev. Lett. 94, 065003 (2005).

[13] G. Tanner and N. Sondergaard, J. Phys. A 40, 443 (2007).

[14] F. Mortessagne, O. Legrand, and D. Sornette, Chaos 3, 529 (1993).

[15] E. G. Altmann, Phys. Rev. A 79, 013830 (2009).

[16] C. P. Dettmann, G. V. Morozov, M. Sieber, and H. Waalkens, Phys. Rev. A 80, 063813 (2009).

[17] J. Schneider, J. Schmalzl, and Tamás Tél, Chaos 17, 033115 (2007).

[18] M. Moshinsky, Phys. Rev. 88, 625 (1952).

[19] A. Goussev, Phys. Rev. A 85, 013626 (2012).

[20] A. Goussev, Phys. Rev. A 87, 053621 (2013).

[21] V. Milner, J. L. Hanssen, W. C. Campbell, and M. G. Raizen, Phys. Rev. Lett. 86, 1514 (2001).
[22] N. Friedman, A. Kaplan, D. Carasso, and N. Davidson, Phys. Rev. Lett. 86, 1518 (2001).

[23] K. A. Mitchell and D. A. Steck, Phys. Rev. A 76, 031403 (2007).

[24] K. A. Mitchell and B. Ilan, Phys. Rev. A 80, 043406 (2009).

[25] R. D. Vilela and A. E. Motter, Phys. Rev. Lett. 99, 264101 (2007).

[26] G. Cristadoro, G. Knight, and M. D. Esposti, J. Phys. A 46, 272001 (2013).

[27] P. Glendinning and N. Sidorov, Ergod. Theor. Dyn. Syst., doi:10.1017/etds.2013.98.

[28] R. A. Barrera, arXiv:1306.2054 (2013).

[29] L. A. Bunimovich and B. Webb, arXiv:1211.4617 (2013).

[30] G. Knight, O. Georgiou, C. P. Dettmann, and R. Klages, Chaos 22, 023132 (2012).

[31] O. Georgiou, C. P. Dettmann, and E. G. Altmann, Chaos 22, 043115 (2012).

[32] C. P. Dettmann, Nonlinearity 26, 307 (2013).

[33] V. Paar and N. Pavin, Phys. Rev. E 55, 4112 (1997).

[34] M. F. Demers and L. S. Young, Nonlinearity 19, 377 (2006).

[35] S. M. Ulam, A Collection of Mathematical Problems, Interscience Tracts in Pure and Applied Mathematics, no. 8 (Interscience Publishers, New York, 1960).

[36] W. Bahsoun, Nonlinearity 19, 2529 (2006).

[37] W. Bahsoun, C. Bose, and Y. Duan, arXiv:1301.4033 (2013).

[38] G. Froyland, Nonlinearity 12, 79 (1999).

[39] G. Froyland, Nonlinearity 12, 1029 (1999).

[40] W. Bahsoun and C. Bose, Nonlinear Anal.: Theor. Meth. Appl. 74, 4481 (2011).

[41] M. F. Demers, P. Wright, and L.-S. Young, Commun. Math. Phys. 294, 353 (2010).

[42] A. Nemeth and P. Szepfalusy, Phys. Rev. E 52, 1544 (1995).

[43] H. Lustfeld and P. Szepfalusy, Phys. Rev. E 53, 5882 (1996). 